\title{
Satellite Observations of Strong Balmer Alpha Atmospheric Emissions around the Magnetic Equator
}

\author{
A. C. Levasseur and J. E. Blamont \\ Service d'Aéronomie du CNRS, Verrières le Buisson, France
}

\begin{abstract}
Balmer $\alpha$ emissions of intensity larger than three times the nominal value have been recorded during five different nights at geomagnetic latitudes inferior to $\pm 22^{\circ}$. They are correlated with sudden impulses in magnetograms.
\end{abstract}

Description of Balmer $\alpha$ Experiment on D2A

Scientific abjective. The objective of this experiment is to study the density and distribution of atomic hydrogen in the earth's outer atmosphere by measuring the intensity of Balmer $\alpha$ emission. This emission, which has an intensity of a few rayleighs, was discovered in 1958 by ground-based observations made by Prokundina [1959], and then by Fishkova and Markova [1960], and Sheglov [1964]; it has also since been observed by Ingham [1962, 1968], Tinsley [1967, 1970] and Levasseur [1969]. Tinsley and Meier [1971] have conducted a systematic study of the emission since 1965 .

However, such ground measurements are unable to provide a worldwide global survey all through the year. Moreover, some of them are strongly contaminated by night sky emissions and a continuous background. The night sky emissions are mainly due to $\mathrm{OH}$, the emission of which occurs between the altitudes of 80 and $120 \mathrm{~km}$. The continuous background is made up of three components, star light, zodiacal light, and airglow continuum, which originates from about the same altitudes as $\mathrm{OH}$.

The ' $\mathrm{H} \alpha$ ' experiment on D2A was therefore designed as a two-channel photometer with 20-A filters, which would simultaneously measure the light emission originating above the altitude of $150 \mathrm{~km}$ at $6563 \mathrm{~A}$ and $6530 \mathrm{~A}$. The Balmer $\alpha$ emission would be determined by subtracting the 6530-A emission from the 6563-A emission.

Spacecraft. 'D2A 'Tournesol', launched on April 15, 1971, from Kourou (French Guiana) is a 96-kg solar-oriented spacecraft (Figure 1). The orbit parameters at launch were apogee

Copyright (c) 1973 by the American Geophysical Union.
$700 \mathrm{~km}$, perigee $456 \mathrm{~km}$, and inclination $45^{\circ}$. The satellite is a cylinder whose spin axis is kept parallel to the sun spacecraft direction by an active stabilization system with an accuracy of a few minutes. The satellite rotates around this axis with a 60-sec period. Two types of optical experiments are carried by $\mathrm{D} 2 \mathrm{~A}$. The first kind has its optical axis parallel to the rotation axis, and the second has its optical axis perpendicular to the rotation axis. The $\mathrm{H} \alpha$ photometer is of the second type and therefore every $60 \mathrm{sec}$ it scans the atmosphere. During a rotation the point of closest approach first coincides with the spacecraft, then goes down through the layers of the atmosphere, reaches the earth, and again goes up through the atmosphere. During some rotations near local midnight the optical axis may not reach the earth, as can be seen in Figure 2, which is made in the plane of the orbit, and it depends on the relative positions of the sun and of the perigee.

Optics and electronics. The photometer (Figure 3) comprises one lens, two filters, and a dualcathode photomultiplier (RTC type $450 \mathrm{FH}$ ). The lens (diameter $=100 \mathrm{~mm}$, focal length $=$ $500 \mathrm{~mm}$ ) projects an image of the sky in its focal plane. The flat mirror is used to rotate the beam by $92^{\circ}$ because of the spacecraft's geometrical constraints. Placed before the focal plane, a shutter allows measurements of the dark current and on board calibrations provided by a small light source. When actuated by the photodiode, the shutter protects the photomultiplier against strong signals such as sunlit earth emission. In the focal plane, two interference filters are placed in front of a dual-cathode photomultiplier; the wavelengths of peak transmission for these filters are $6563 \mathrm{~A}$ and $6530 \mathrm{~A}$. Thus the incident 


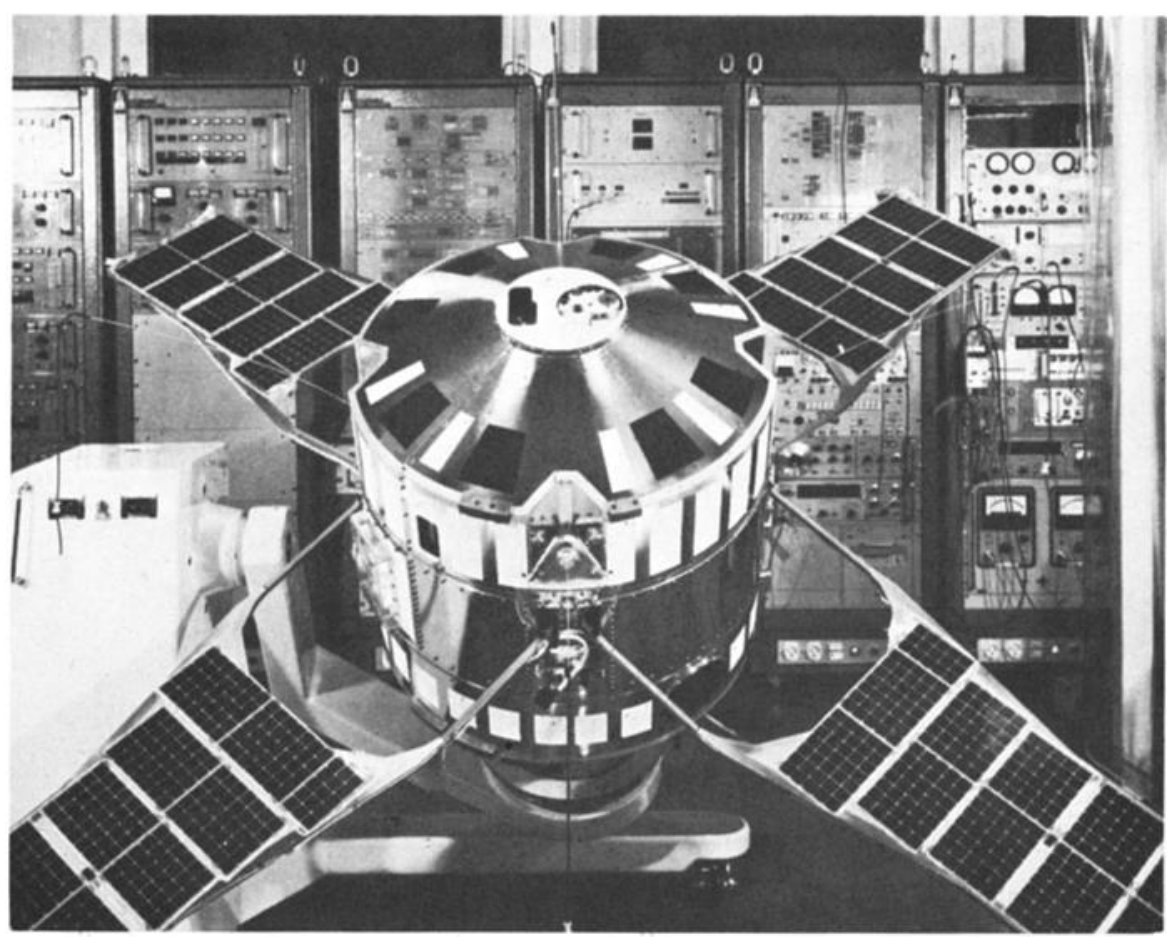

Fig. 1. Photo of the D2A spacecraft.

beam is split into two beams, one at Balmer $\alpha$, and the other one at Balmer $\alpha-33 \mathrm{~A}$. Each of these beams hits a separate photocathode.

The photomultiplier has two cathodes but only one multiplier system, and there is only one amplifier for the two channels. A 7/8-sec observation is provided simultaneously for both wavelengths every 2 sec. The optical axis therefore rotates about the spacecraft by $5^{\circ} 15^{\prime}$ during each measurement. The signal is processed by a pulse-counting technique with threshold discrimination and a floating point counter and transmitted via pulse count modulation telemetry. The dark current is measured once every 256 sec when the shutter is closed for $7 / 8 \mathrm{sec}$ and during half the time of the calibration sequences, which are initiated from ground command.

To summarize, the photometer measures simultaneously during $7 / 8$ sec the intensity of the Balmer $\alpha$ emission $I_{H}$ on which is superimposed the intensity of the background emission at $6563 \mathrm{~A}$ and the intensity of the background emission at 6530 A $I_{0}$.

Instrumental performances. The field of view for each channel would be $1^{\circ} 20^{\prime} \times 2^{\circ} 40^{\prime}$ on a nonspinning spacecraft. However, during the measurement time of $7 / 8$ sec the satellite rotates, and it is therefore $6^{\circ} 35^{\prime} \times 2^{\circ} 40^{\prime}$.

The spectral response is illustrated at the average temperature of the inflight experiment $\left(10^{\circ} \mathrm{C}\right)$ by Figure 4 . The bandwidths can be taken safely as about $20 \mathrm{~A}$.

The response of the instrument to five secondary sources was determined. These sources (white Osram flat tungsten ribbon lamps) had been calibrated carefully against primary standards from the Physikalishes Institut in Berlin. The estimated preliminary uncertainty in the absolute value was $<50 \%$. This uncertainty has been reduced by observations in the orbit of the Gum Nebula and of the zodiacal light by the instrument. The photometer yields $(75 \pm 3)$ pulses for 1 rayleigh of Balmer $\alpha$ light, whereas the dark current is $<60$ pulses/sec. The linearity was found to be better than $1 \%$ over the range $0.5-100$ rayleighs.

The internal polarization factor of the photometer is $<1 \%$. This point is not to be neglected because the Balmer $\alpha$ emission is polarized, as is shown by the observations we have performed at the Haute-Provence Observatory (1969). The 
Levasseur and Blamont: Equatorial Balmer Alpha Emisgions

3883

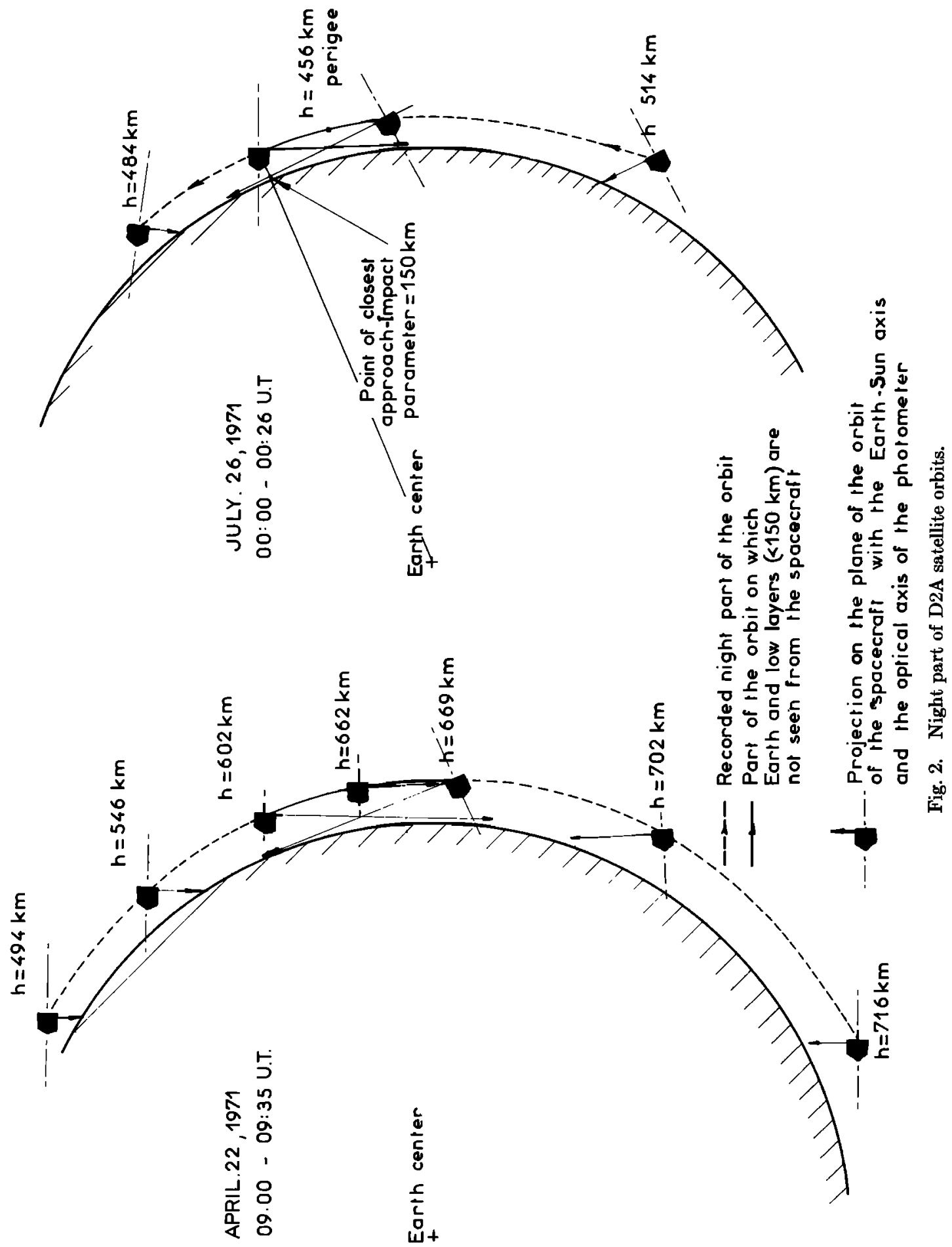




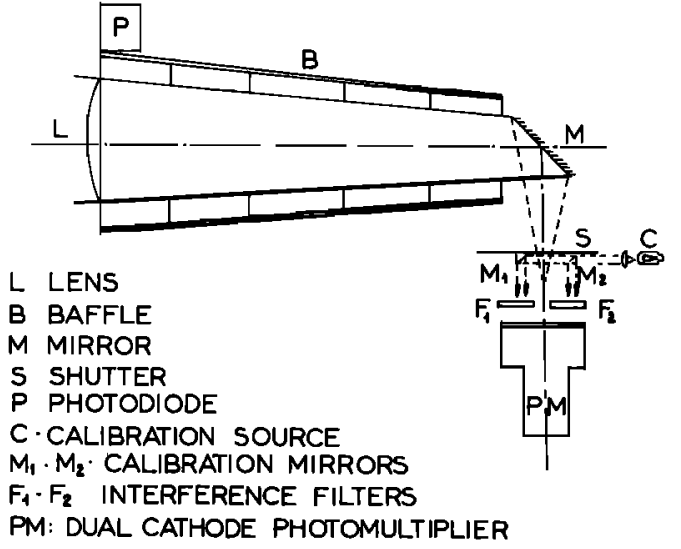

Fig. 3. Optical schematic of the $\mathrm{H} \alpha$ photometer on satellite D2A.

effect of the earth's magnetic field was found to be negligible due to the photomultiplier magnetic shielding.

In fight performances. The $\mathrm{H} \alpha$ photometer was turned on by ground command on April 19, 1971 , and is still operating after 19 months.

The ratio of the calibration signals in the two channels as measured in orbit is identical to the laboratory value, and the absolute values of the calibration signals are also unchanged.

During the day part of the orbit the stray light is strong, and the shutter remains closed. During the night part the dark current is measured every $4 \mathrm{~min}$. It is about 60 pulses/sec during most orbits and reaches 100-140 pulses/sec during the very short night orbits. We have compared it with the dark current that is expected from laboratory values at the same temperature. There

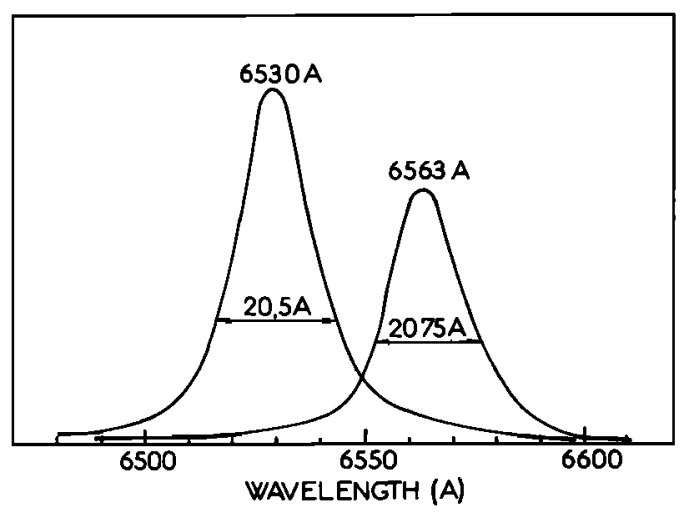

Fig. 4. Transmission curves for the two channels of the $\mathrm{H}_{\alpha}$ photometer. is a good agreement, except for the measurements made above the South Atlantic anomaly (where it may be higher than 1000 pulses), and one unique measurement, on April 19, 1972, above South Africa (768-656 pulses).

\section{Data Reduction}

The data reduction has already been performed for 33 nights chosen between April 1971 and August 1972. The purpose of this paper is not to describe all the results on Balmer $\alpha$ geocoronal or nebular emission and on zodiacal light, they are reported separately [Blamont and Levasseur, 1971; Blamont et al., 1971; Levasseur, 1972]; but we shall summarize them in order to explain by which way we give evidence of an intense Balmer $\alpha$ emission around the magnetic equator.

Data analysis. The Balmer $\alpha$ emissions may be telluric, and more particularly, geocoronal (scattering of the solar Lyman beta line by the atmospheric hydrogen and deexcitation). During the 1-min rotation of the spacecraft, as can be seen on Figure 5, different layers of the atmosphere are successively scanned; the impact parameter decreases from the value of the satellite altitude to a lower value, which may be zero, and then goes up from that value to the satellite altitude. They also may be astronomical, originating in H II regions. Every $60 \mathrm{sec}$ the optical axis traces out a great circle on the celestial sphere. This circle rotates across the sky at $1^{\circ}$ per day due to the orbital motion of the earth.

Background emissions may be due to the zodiacal cloud, the airglow continuum, and the starlight. The intensity $I_{z}$ of the zodiacal light is always measured for a $90^{\circ}$ solar elongation, because the optical axis of the experiment is perpendicular to the sun-earth axis.

Actually, we have only taken into account the measurements for which: first, the impact parameter remains above $150 \mathrm{~km}$; consequently, the measured emission originates above the altitude of $150 \mathrm{~km}$. There is no airglow continuum in the analyzed data, and the contamination by any hydroxyl line is avoided. Second, there is no bright star, or part of the Milky Way in the field of view, and the galactic latitude remains out of $\mathrm{a} \pm 30^{\circ}$ bracket; the only stellar contribution to the background emissions therefore comes from integrated starlight.

Zodiacal light. The study of the zodiacal light 


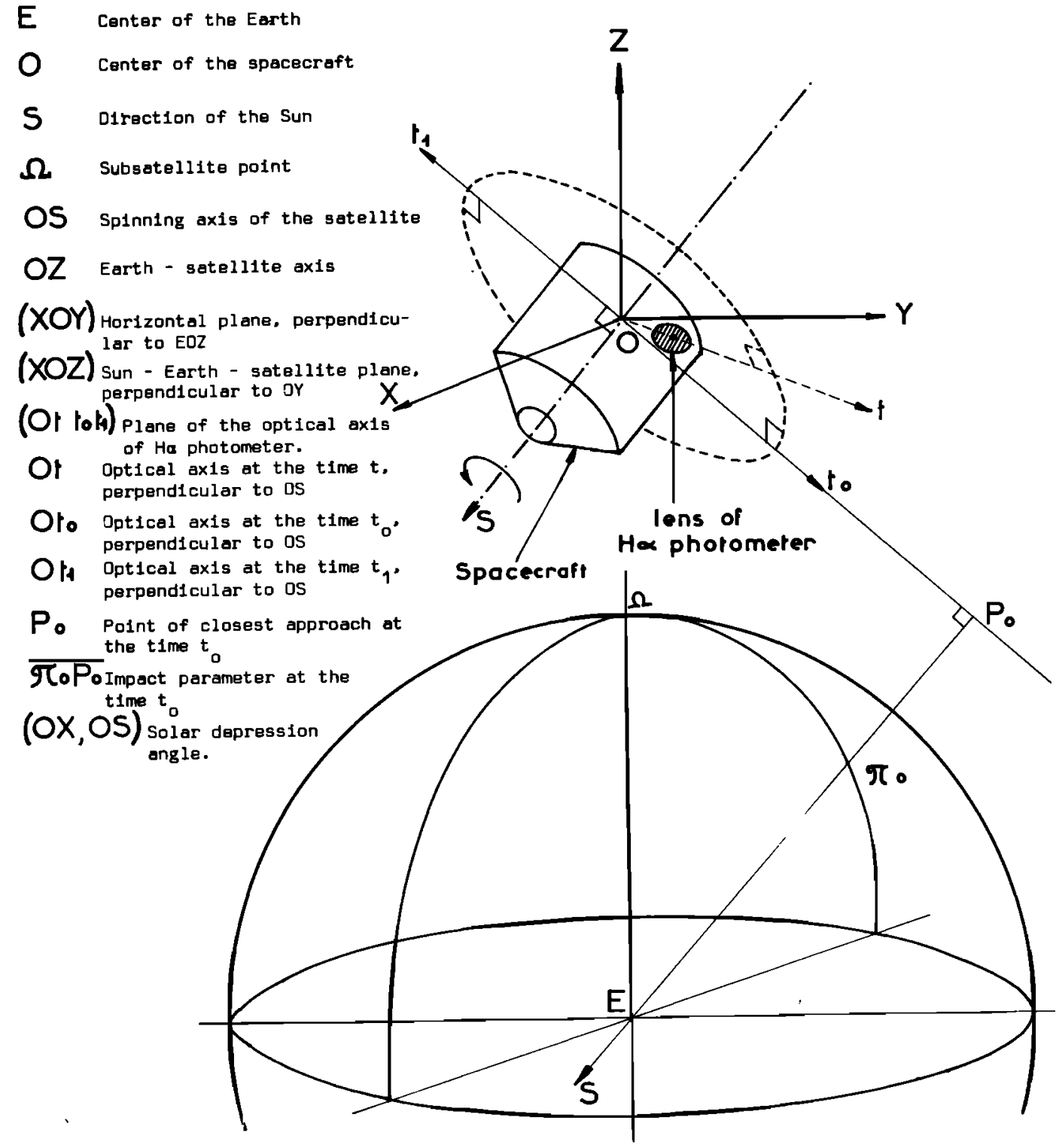

Fig. 5. D2A spacecraft attitude.

is made through measurements on the $6530-\mathrm{A}$ channel.

The strength of the 6530-A signal $N_{0}$ is about $1000 \mathrm{pulses} / \mathrm{sec}$; the dark current $n_{0}$ remains under 100 pulses/sec when the spacecraft does not pass through the South Atlantic anomaly and the signal-to-noise ratio is $>10$. If $C_{0}$ is the calibration coefficient on channel $6530 \mathrm{~A}$ and $\Delta \lambda_{0}$ the bandwidth, the intensity is computed in $\mathrm{R} / \mathrm{A}$ by the following formula:

$$
I_{0}=\left(N_{0}-n_{0}\right)\left(C_{0} / \Delta \lambda_{0}\right)
$$

This signal is made up of zodiacal light $I_{z}$ and of integrated starlight. For high-galactic latitudes $\left|b_{I I}\right|>30^{\circ}$, and any galactic longitude $l_{I I}$, the integrated starlight $I_{s}\left(l_{I I}, b_{I I}\right)$ taken from the tables of Smith et al. [1965] remains smaller than $0.2 \mathrm{R} / \mathrm{A}$. We therefore identify $I_{z}$ as

$$
I_{z}=\left(N_{0}-n_{0}\right)\left(C_{0} / \Delta \lambda_{0}\right)-I_{s}\left(l_{I I}, b_{I I}\right)
$$


The ecliptic latitude rotates by $360^{\circ}$ each minute (Figure 6). For $0^{\circ}$ ecliptic latitude, $I_{z}$ is in a $0.9-$ to $1.2-\mathrm{R} / \mathrm{A}$ bracket, and for $90^{\circ}$ ecliptic latitude, it is approximately $0.5 \pm 0.1$ $\mathrm{R} / \mathrm{A}$. These results are perfectly consistent with ground measurements and give us confidence in our absolute calibration.

Geocoronal emission. The study is made through measurements on the 6563-A channel. Only those measurements, which satisfied the following conditions, were taken into account: neither bright stars nor $\mathrm{H}$ II regions in the field of view, galactic latitude larger than $30^{\circ}$, impact parameter higher than $150 \mathrm{~km}$, and dark current $n_{1}$ at its normal rate. The strength of the $6563-\mathrm{A}$ $N_{1}$ signal is about 1200 pulses/sec. It is made up of zodiacal light, integrated starlight, and telluric Balmer $\alpha$ emissions on which is superimposed $n_{1}$. The Balmer $\alpha$ intensity $I_{H}$ is obtained by subtracting from the total intensity on the channel (calibration coefficient $C_{1}$, bandwidth $\Delta \lambda_{1}$ ) the intensity of zodiacal and integrated starlight on that channel. The zodiacal light, as the average integrated starlight, has a solar-like spectrum, which shows Balmer $\alpha$ in absorption, and the intensity decreases slowly with the wavelength $f(\lambda)$. From the results previously published by Evans [1940] and David [1961], we computed the equivalent width $W$ of the Fraunhofer Balmer $\alpha$ feature in integrated sunlight and the slightly smaller than 1 ratio of $f\left(\lambda_{1}\right)$ to $f\left(\lambda_{0}\right)$. We deduced that, if $I_{0} \Delta \lambda_{0}$ rayleighs are observed on channel $6530 \mathrm{~A}$, the contribution of zodiacal light and integrated starlight on channel $6563 \mathrm{~A}$ must be

$$
I_{0}\left(\Delta \lambda_{1}-W\right)\left[f\left(\lambda_{1}\right) / f\left(\lambda_{0}\right)\right]
$$

Then

$$
I_{H}=\left[\left(N_{1}-n_{1}\right)-\left(N_{0}-n_{0}\right) P\right] C_{1}
$$

where

$$
P=\frac{C_{0}}{\Delta \lambda_{0}} \frac{\Delta \lambda_{1}-W}{C_{1}} \frac{f\left(\lambda_{1}\right)}{f\left(\lambda_{0}\right)}=0.896 \pm 0.016
$$

It was found that (Figure 7) $I_{H}$ is significantly lower by a factor of 2 than that expected from ground-obtained data and remains around 2 rayleighs at midnight. The morning intensity is always greater than the evening intensity for the same depression angle and direction. The geocoronal intensities have been compared with intensities predicted by radiative transfer. Our results are in good agreement with current hydrogen numerical models [Chamberlain, 1963; Vidal-Madjar and Bertaux, 1972]. Preliminary results have been presented at the 1972 Cospar meeting.

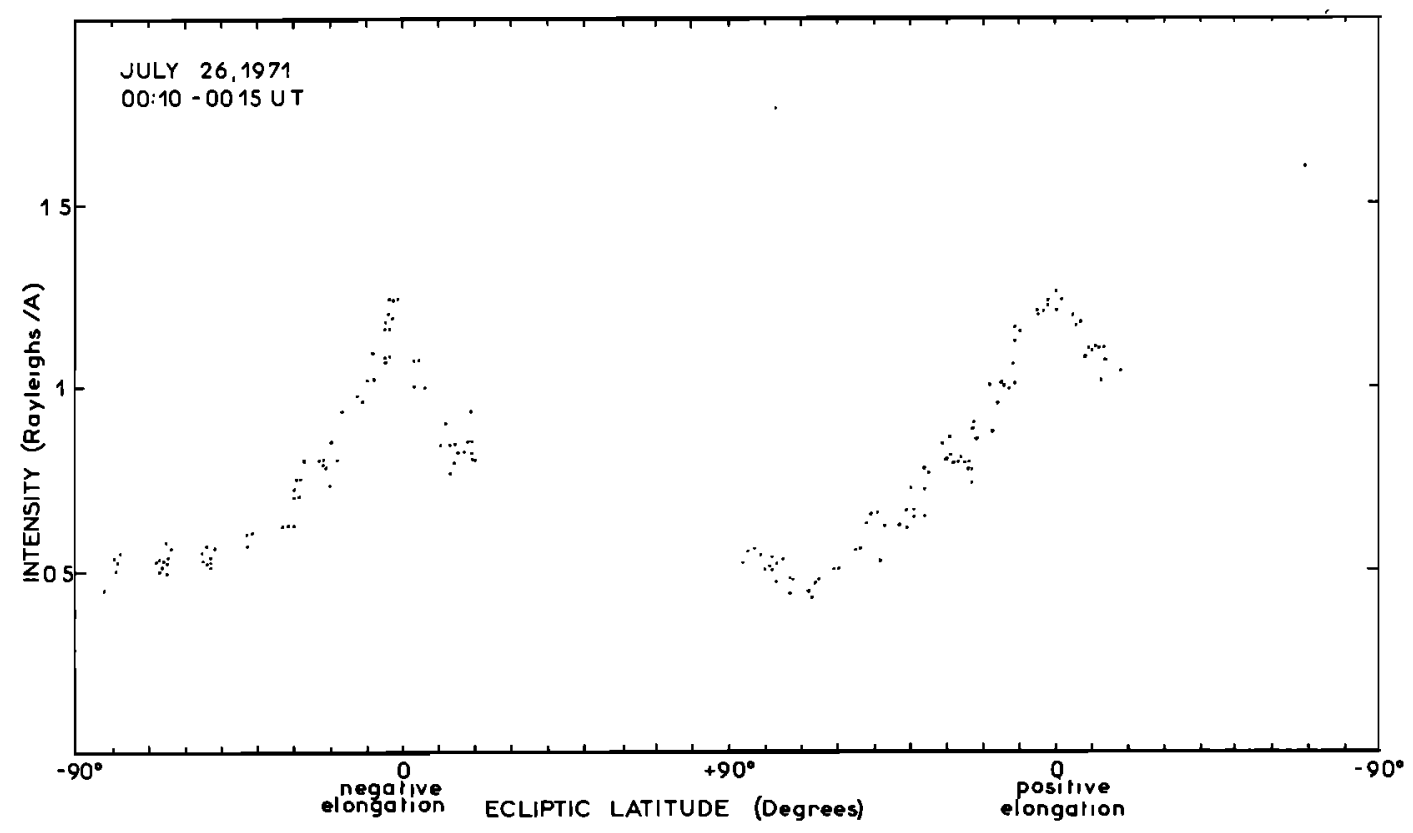

Fig. 6. Zodiacal light intensity at 6530 A versis ecliptic latilude $\left(90^{\circ}\right.$ elongation). 


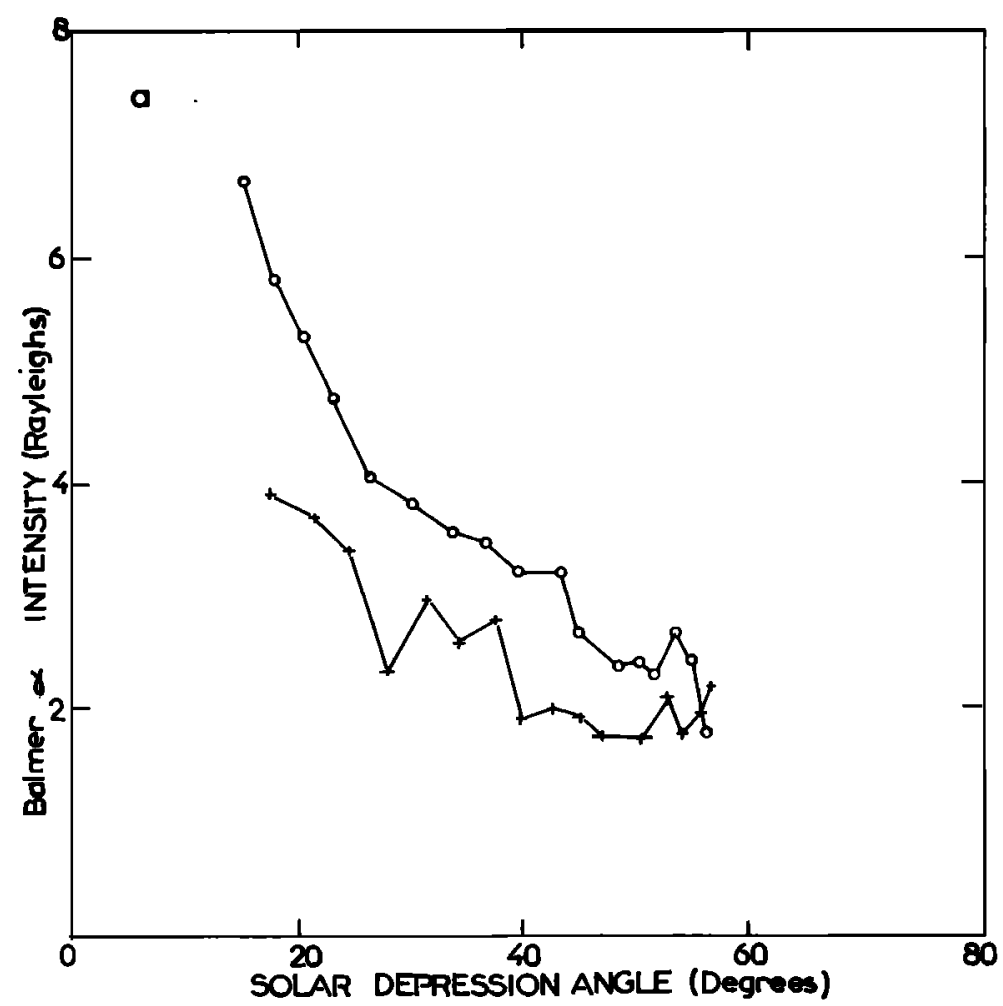

Fig. 7a. Average brightness of the geocoronal $\mathrm{H} \alpha$ emission perpendicular to the sun versus solar depression angle for the orbit on April 20, 1971. For the measurements taken into account the zenith distance was greater than $45^{\circ}$, and its average value for both orbits was equal to $78^{\circ}$. Data obtained before local midnight (crosses) and data obtained after local midnight (circles) are shown.

Nebulae. When an observation is performed in the direction of gaseous nebulae with important emission in Balmer $\alpha$ (as Cygnus, Orion, Crab, Ophiuchus, and Gum Nebula), $I_{H}$ is very strong, and remains between 5 and 60 rayleighs. It was thus found immediately after the spacecraft was launched that the Gum Nebula was recorded as a much more intense feature than the geocoronal emission. If $I_{G}$ represents the average geocoronal emission for the same local time and direction, then $I_{H}-I_{G}$ is the contribution of the nebular source.

A complete map of the sky is obtained with 6 months of data. The intensities recorded in the Gum Nebula are in good agreement with the results of Johnson [1960]. The periodical observation of intense nebulae provides a good check of the photometer stability, and we conclude that the instrument provides accurate and reliable absolute measurements of the $\mathrm{H} \alpha$ emission.

\section{INTERTropical BaLmer $\alpha$ Emission IN the} LOWER THERMOSPHERE

We have said that, when there is no nebula in the field of view, the measured $I_{H}$ is similar to the computed one. However, there were definitely exceptions on five different nights (April 21, 1971; June 23, 1971; September 14, 1971; and April 19 and 20, 1972). A strong intertropical Balmer $\alpha$ emission was observed in the lower thermosphere; the point of closest approach altitude was under $218 \mathrm{~km}$.

Experimental evidence. The events were found after a systematic search in the analyzed part of the data (33 days) with the following conditions on the observations. First, as in all our reduced data, to avoid stray emission the impact parameter remains above $150 \mathrm{~km}$, there are neither bright stars nor nebulae in the field of view, and the galactic latitude is $>30^{\circ}$. Second, to limit the geocoronal intensity the solar depression angle 


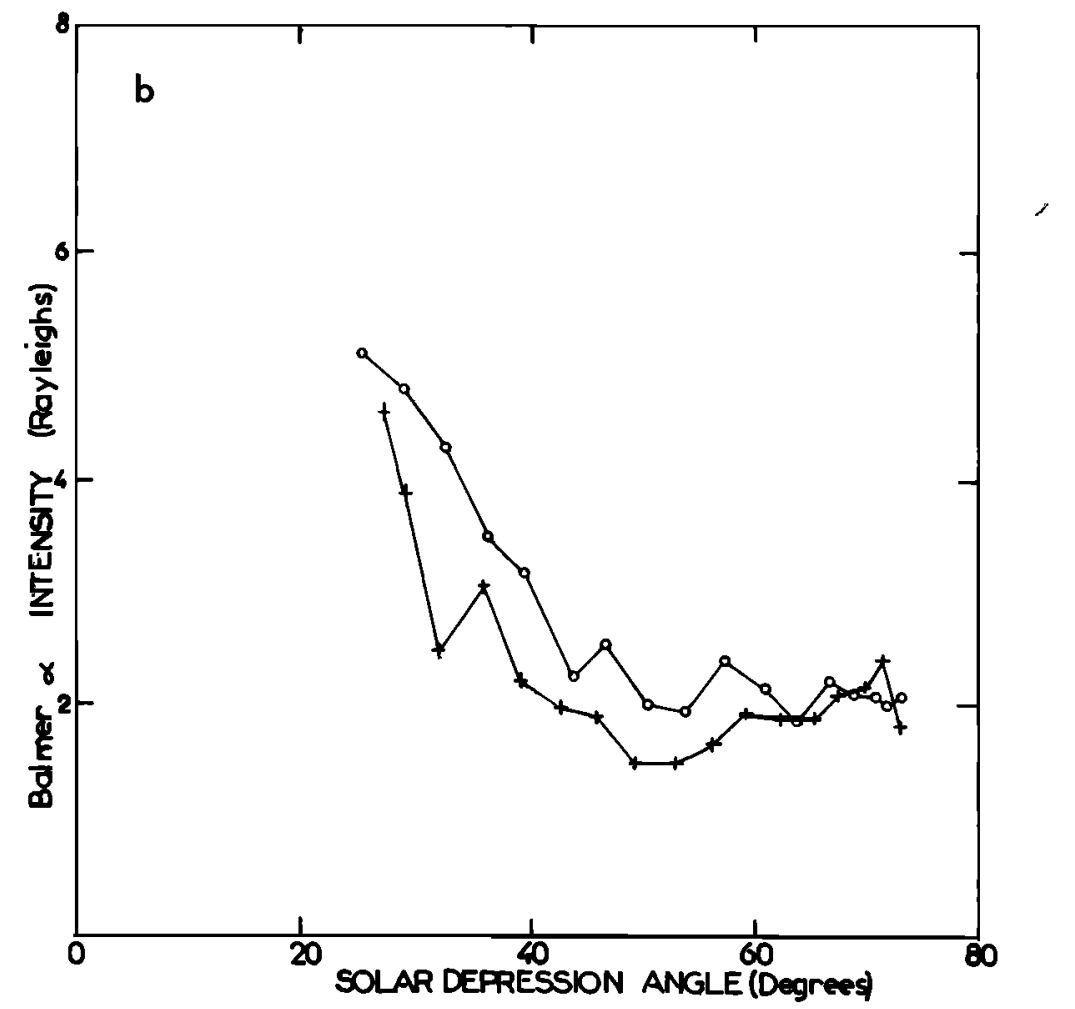

Fig. 7b. Average brightness of the geocoronal $\mathrm{H} \alpha$ emission perpendicular to the sun versus solar depression angle for orbit on April 22, 1971. For the measurements taken into account the zenith distance was greater than $45^{\circ}$, and its average value for both orbits was equal to $78^{\circ}$. Data obtained before local midnight (crosses) and data obtained after local midnight (circles) are shown.

is $>30^{\circ}$ and $I_{G}$ does not reach 5 rayleighs. Third, the satellite is outside the South Atlantic anomaly, so that the dark current has its regular rate of counts per second.

The observed Balmer $\alpha$ emission in these conditions was found to be three times greater than the nominal one on five different days during which 15 measurements of abnormal intensity were obtained. They could even emerge from rough data without any use of a numerical model; whenever the experiment observes geocoronal Balmer $\alpha$ for a solar depression angle that is $>30^{\circ}\left(1.5 \leq I_{G} \leq 5\right.$ rayleighs $)$ and zodiacal light $\left(0.5 \mathrm{R} / \mathrm{A} \leq I_{z} \leq 1.2 \mathrm{R} / \mathrm{A}\right)$, the ratio $N_{1} / N_{0}$ always remains below 1.15 . On the opposite, for the unusual measurements, the ratio was at least 1.3.

During those five days (April 21, 1971; June 23, 1971; September 14, 1971; and April 19 and 20,1972$)$ the other measurements performed during the same spacecraft rotation were nominal. The observed Balmer $\alpha$ and zodiacal light emissions were at that time consistent with previous measurements and numerical models. In addition, the unusual measurements were not only obtained once in a certain direction, the intensity of the emission originating from roughly the same direction was measured and found to be abnormally great for at least two different and consecutive satellite scans. We hold that, when the same enhancement is observed at least two times at a 1-min interval in a single channel, it cannot originate in data transmission anomalies or dark current fluctuations.

We therefore designate an event the observation of a Balmer $\alpha$ emission, whose intensity is at least three times larger than the average geocoronal intensity observed when stray emissions are much reduced and when the measured zodiacal light is nominal. Some parameters relevant to these five events are in Table 1. Intensity. For the events the Balmer $\alpha$ 
TABLE 1. Parameters Relevant to Measurements in the Lower Thermosphere*

\begin{tabular}{|c|c|c|c|c|c|c|c|c|c|}
\hline \multirow[b]{3}{*}{$N_{1} / N_{0}$ pulses } & \multirow{3}{*}{$\begin{array}{c}I_{H}, \\
\text { ray- } \\
\text { leighs }\end{array}$} & \multirow{3}{*}{$\begin{array}{c}I_{H}- \\
I_{G} \\
\text { ray- } \\
\text { leighs }\end{array}$} & \multirow{3}{*}{$\begin{array}{c}I_{Z} \\
\mathrm{R} / \mathrm{A}\end{array}$} & \multirow[b]{3}{*}{$\begin{array}{c}\text { Excess, } \\
\text { R/A }\end{array}$} & \multirow[b]{3}{*}{$\begin{array}{l}\text { Altitude, } \\
\text { km }\end{array}$} & \multicolumn{4}{|c|}{ Impact Parameter } \\
\hline & & & & & & \multicolumn{2}{|c|}{ Geographic } & \multicolumn{2}{|c|}{ Geomagnetic } \\
\hline & & & & & & $\begin{array}{c}\text { Latitude, } \\
\text { degrees }\end{array}$ & $\begin{array}{c}\text { Longitude, } \\
\text { degrees }\end{array}$ & $\begin{array}{c}\text { Latitude, } \\
\text { degrees }\end{array}$ & $\begin{array}{c}\text { Longitude, } \\
\text { degrees }\end{array}$ \\
\hline \multicolumn{10}{|c|}{ April 21, 1971, $0740 \mathrm{UT}$} \\
\hline$\frac{3168}{1344}=2.4$ & 25 & 22 & 0.4 & $o$ & $150-303$ & -23 & 212 & -21 & -73 \\
\hline$\frac{2080}{2080}=1.0$ & 1 & $\mathbf{0}$ & 1.2 & 0 & $210-353$ & -47 & 252 & -38 & -32 \\
\hline$\frac{4960}{3744}=1.3$ & $\mathscr{2 2}$ & 19 & 0.4 & 0 & $163-183$ & -14 & 243 & -6 & -46 \\
\hline$\frac{1376}{1184}=1.2$ & 2 & $\mathbf{0}$ & 0.5 & 0 & $212-340$ & 13 & 276 & 24 & -15 \\
\hline$\frac{\mathbf{1 3 4 4}}{\mathbf{1 2 4 8}}=1.1$ & 3 & 0 & 0.6 & 0 & $190-346$ & 28 & 286 & 39 & -1 \\
\hline \multicolumn{10}{|c|}{ June 23, 1971, 0330 UT } \\
\hline$\frac{1952}{992}=2.0$ & 14 & 10 & 0.5 & 0 & $171-296$ & 1 & 356 & 5 & 67 \\
\hline$\frac{2936}{976}=2.4$ & 19 & 15 & 0.5 & $o$ & $164-296$ & 6 & 359 & 10 & 70 \\
\hline$\frac{2592}{944}=2.7$ & 23 & 20 & 0.5 & 0 & $163-301$ & $\gamma$ & 3 & 10 & 74 \\
\hline$\frac{1408}{1344}=1.1$ & 3 & $\mathbf{0}$ & 0.5 & 0 & $182-316$ & 32 & 340 & 38 & 56 \\
\hline$\frac{2400}{1024}=2.3$ & 19 & 15 & 0.5 & 0 & $168-310$ & 10 & 6 & $1 \mathscr{2}$ & 78 \\
\hline \multicolumn{10}{|c|}{ September 14, 1971, $0600 \mathrm{UT}$} \\
\hline$\frac{1440}{1248}=1.1$ & 4 & $\mathbf{0}$ & 0.7 & 0.2 & $219-326$ & 24 & 11 & 26 & 87 \\
\hline$\frac{3616}{1568}=1.9$ & 27 & 21 & 0.9 & 0.3 & $161-303$ & -4 & 313 & 6 & 23 \\
\hline$\frac{1568}{1632}=1.0$ & 2 & $\mathbf{0}$ & 1.0 & 0.3 & $197-334$ & 28 & 303 & 39 & 15 \\
\hline$\frac{3794}{1856}=2.0$ & 28 & 24 & 0.9 & 0.3 & $150-278$ & 1 & 910 & 12 & 20 \\
\hline \multicolumn{10}{|c|}{ April 19, 1972, First Orbit, 1740 UT } \\
\hline$\frac{3616}{2144}=1.7$ & 20 & 18 & 1.2 & $o$ & $155-381$ & -19 & 32 & -21 & 98 \\
\hline$\frac{2080}{2016}=1.0$ & 3 & 0 & 1.0 & 0 & $165-387$ & -61 & 49 & -64 & 98 \\
\hline
\end{tabular}


TABLE 1. (continued)

\begin{tabular}{|c|c|c|c|c|c|c|c|c|c|}
\hline \multirow[b]{3}{*}{$N_{1} / N_{0}$ pulses } & \multirow{3}{*}{$\begin{array}{c}I_{H} \\
\text { ray- } \\
\text { leighs }\end{array}$} & \multirow{3}{*}{$\begin{array}{c}I_{H}- \\
I_{G} \\
\text { ray- } \\
\text { leighs }\end{array}$} & \multirow[b]{3}{*}{$\begin{array}{c}I_{Z} \\
\mathrm{R} / \mathrm{A}\end{array}$} & \multirow[b]{3}{*}{$\begin{array}{l}\text { Excess, } \\
\text { R/A }\end{array}$} & \multirow[b]{3}{*}{$\begin{array}{l}\text { Altitude, } \\
\text { km }\end{array}$} & \multicolumn{4}{|c|}{ Impact Parameter } \\
\hline & & & & & & \multicolumn{2}{|c|}{ Geographic } & \multicolumn{2}{|c|}{ Geomagnetic } \\
\hline & & & & & & $\begin{array}{l}\text { Latitude, } \\
\text { degrees }\end{array}$ & $\begin{array}{c}\text { Longitude, } \\
\text { degrees }\end{array}$ & $\begin{array}{c}\text { Latitude, } \\
\text { degrees }\end{array}$ & $\begin{array}{c}\text { Longitude, } \\
\text { degrees }\end{array}$ \\
\hline$\frac{2836}{1600}=1.5$ & 10 & 8 & 0.8 & 0 & $218-406$ & -15 & 43 & -19 & 110 \\
\hline \multicolumn{10}{|c|}{ April 19, 1972, Second Orbit, 1920 UT $\dagger$} \\
\hline$\frac{3232}{3552}=0.9$ & 2 & 0 & 1.8 & 0.8 & $162-357$ & -52 & 40 & -55 & 95 \\
\hline$\frac{3808}{2976}=1.3$ & 13 & 10 & 1.4 & 0.6 & $168-352$ & -11 & 24 & -12 & $9 \mathscr{2}$ \\
\hline$\frac{4576}{3960}=1.4$ & 18 & 16 & 1.7 & 0.9 & $163-923$ & -7 & 32 & -10 & 101 \\
\hline$\frac{3960}{2592}=1.3$ & 11 & 9 & 1.1 & 0.6 & $162-275$ & -2 & 46 & -7 & 115 \\
\hline$\frac{2336}{2528}=0.9$ & 1 & 0 & 1.2 & 0.1 & $192-285$ & -30 & 6 & -36 & 125 \\
\hline \multicolumn{10}{|c|}{ April 20, 1972, $0330 \mathrm{UT}$} \\
\hline$\frac{2656}{2016}=1.3$ & 22 & 20 & 0.5 & 0 & $153-194$ & -9 & 313 & $\mathscr{Z}$ & 23 \\
\hline$\frac{5344}{3808}=1.4$ & 25 & 22 & 2.0 & 0.8 & $200-269$ & 0 & 294 & 11 & 4 \\
\hline$\frac{2208}{2144}=1.0$ & 3 & 0 & 1.2 & 0 & $210-368$ & 27 & 345 & 33 & 61 \\
\hline
\end{tabular}

* Italics indicate events.

$\dagger$ Increase of dark current.

intensity $I_{H}$ is in a 10- to 25-rayleigh bracket; the difference between $I_{H}$ and the standard geocoronal intensity $I_{G}$ has an average value of 17 rayleighs and has been found to reach 24 rayleighs.

The only measurements for which we have not a very good confidence in the computed $\left(I_{H}-I_{G}\right)$ were performed during the second orbit of April 19, 1972, at longitude $31^{\circ} \mathrm{E}$, latitude $-27^{\circ}$, and altitude $680 \mathrm{~km}$. The dark current was unusually high then. The difference between the measured zodiacal light intensity $I_{z}$ on channel $6530 \mathrm{~A}$ and the expected (from ecliptic elongation) zodiacal light intensity was about $0.5 \mathrm{R} / \mathrm{A}$. This may be explained either by the statistical fluctuations of the high dark current or by a Doppler broadened and/or shifted abnormal Balmer $\alpha$ emission. In any case, on the three irregular measurements, $N_{1} / N_{0}$ was $>1.3$ and the observed phenomenon was obviously localized in the vicinity of $6563 \mathrm{~A}$.

Altitude. The two extreme altitudes of the point of closest approach are in Table 1 for all the irregular data; the duration of the measurement and the field of view have been taken into account. Up to now no noticeable increase in the Balmer $\alpha$ emission has been found above $218 \mathrm{~km}$. The events occur in the lower thermosphere, and, as the impact parameter decreases, the irregular Balmer $\alpha$ emission increases.

Latitude. We have searched the data and reduced all the measurements performed under 
$220 \mathrm{~km}$. During the 1-min rotation of the optical axis, the 150- to $220-\mathrm{km}$ layer is at most scanned twice; the restrictive conditions already described, as well as the satellite altitude, limit the number of such data. That is the reason why only 115 lower thermosphere $(150-220 \mathrm{~km})$ observations have been found in the reduced data. They are distributed on 21 nights, and include the irregular observations reported here (Table 2).

For the days on which we have had evidence for an event, two types of low layer measurements. (one before the optical axis reaches the earth, and the other one after) have been performed. One of them coincides with an enhancement of the Balmer $\alpha$ emission; the other one is normal and $I_{H}$ has its predicted intensity. Both are in Table 1. Figure 8 maps all those measurements versus geographic and geomagnetic coordinates; the subsatellite point and the point of closest approach projection on the earth are shown; the impact parameter variations during the 7/8-sec measurement and the excess of Balmer $\alpha$ intensity $\left(I_{H}-I_{G}\right)$ are also shown. This fact demonstrates that the events only occur in low magnetic latitude regions. This may remind one of the photographs of the earth taken on April 21, 1972, with the Carruthers Apollo 16 lunar surface UV camera where a luminous belt almost coincides in time and space with our observation of a Balmer $\alpha$ event [Carruthers, 1972].

It is easy to understand why, up to now, evidence had never been found for such events: the ground observations had been performed in the USSR, the United States, and France. Only four nights of observations had been made in Bolivia by Ingham [1962].

\section{Conclusion: Correlation with Geophysical ACTIVITY}

We have presented some characteristics of the Balmer $\alpha$ emission, which occurs on certain days under $220 \mathrm{~km}$ in equatorial regions. We expect to observe more than five events from the spacecraft, but this paper only reports, with the already reduced data, the present state of our inquiries. From a systematic search in solar geophysical data, we have found a correlation with sudden impulses in the magnetograms given by 10 or more stations. On April 20, 1971, at $1840 \mathrm{UT}$, on June 22, 1971, at $2304 \mathrm{UT}$, on September 13, 1971, at 1338 UT, and on April 18, 1972 , at 0629 UT, i.e., a few hours before the
TABLE 2. Number of Low Layer Measurements Already Reduced

\begin{tabular}{|c|c|c|c|c|}
\hline \multirow[b]{2}{*}{ Date } & \multicolumn{2}{|c|}{$\begin{array}{c}\text { Number of } \\
\text { Standard Lower } \\
\text { Thermosphere } \\
\text { Measurements }\end{array}$} & \multicolumn{2}{|c|}{$\begin{array}{l}\text { Number of } \\
\text { Irregular Lower } \\
\text { Thermosphere } \\
\text { Measurements }\end{array}$} \\
\hline & Total & $\begin{array}{c}\text { Inter- } \\
\text { tropical }\end{array}$ & Total & $\begin{array}{l}\text { Inter- } \\
\text { tropical }\end{array}$ \\
\hline
\end{tabular}

\begin{tabular}{lrrrrr}
\multirow{4}{*}{ April } & \multicolumn{5}{c}{1971 Data } \\
& 20 & 3 & 3 & 0 & 0 \\
& 21 & 3 & 0 & 2 & 2 \\
& 22 & 13 & 12 & 0 & 0 \\
\multirow{4}{*}{ May } & 23 & 11 & 6 & 0 & 0 \\
& 29 & 0 & 0 & 0 & 0 \\
& 4 & 0 & 0 & 0 & 0 \\
& 8 & 0 & 0 & 0 & 0 \\
& 9 & 0 & 0 & 0 & 0 \\
\multirow{4}{*}{ June } & 10 & 0 & 0 & 0 & 0 \\
\multirow{4}{*}{ July } & 17 & 0 & 0 & 0 & 0 \\
\multirow{4}{*}{ September } & 21 & 11 & 7 & 0 & 0 \\
& 23 & 1 & 0 & 4 & 4 \\
& 22 & 11 & 10 & 0 & 0 \\
& 25 & 13 & 10 & 0 & 0 \\
& 1 & 4 & 0 & 0 & 0 \\
& 7 & 0 & 0 & 0 & 0 \\
& 8 & 0 & 0 & 0 & 0 \\
& 9 & 0 & 0 & 0 & 0 \\
& 10 & 0 & 0 & 0 & 0 \\
& 14 & 2 & 0 & 2 & 2
\end{tabular}

\begin{tabular}{|c|c|c|c|c|c|}
\hline \multirow[b]{2}{*}{ April } & \multicolumn{4}{|c|}{ 1972 Data } & \multirow[b]{2}{*}{5} \\
\hline & 19 & 3 & 0 & 5 & \\
\hline & 20 & 1 & $\mathbf{0}$ & 2 & 2 \\
\hline \multirow{11}{*}{ August } & 21 & 0 & $\mathbf{0}$ & 0 & 0 \\
\hline & 22 & 3 & 3 & 0 & 0 \\
\hline & 23 & 6 & 4 & 0 & 0 \\
\hline & 24 & 0 & 0 & 0 & 0 \\
\hline & 25 & 1 & 1 & 0 & 0 \\
\hline & 2 & 2 & 1 & 0 & 0 \\
\hline & 3 & 1 & 1 & 0 & 0 \\
\hline & 4 & 5 & o & 0 & 0 \\
\hline & 5 & 1 & 1 & 0 & 0 \\
\hline & 6 & 1 & 1 & 0 & 0 \\
\hline & 7 & 4 & 4 & 0 & 0 \\
\hline
\end{tabular}

observation of a strong increase in Balmer $\alpha$ telluric emission, equatorial stations recorded sudden impulses in the magnetograms. Such a phenomenon has occurred about 20 times between April 1971 and August 1972. During the period between August 2 and August 7, 1972, no event was observed; at that time, there were worldwide geophysical alerts, but no sudden impulses were found in the magnetograms. When data were available, Balmer $\alpha$ events and sudden impulses were correlated. 


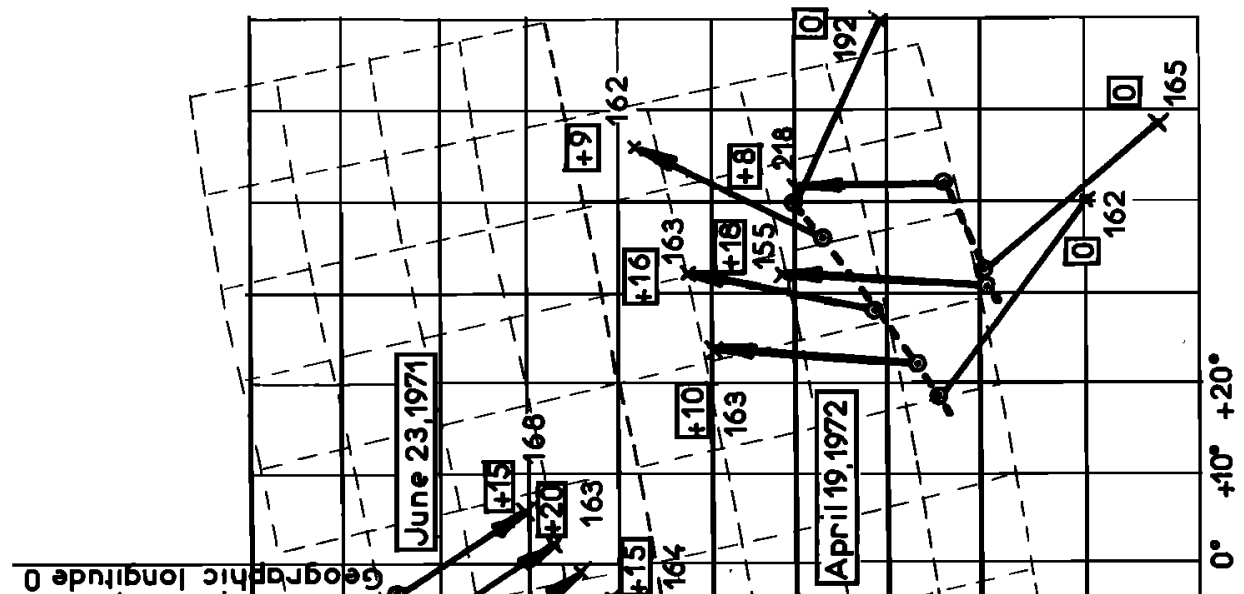

0
0
0

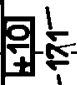

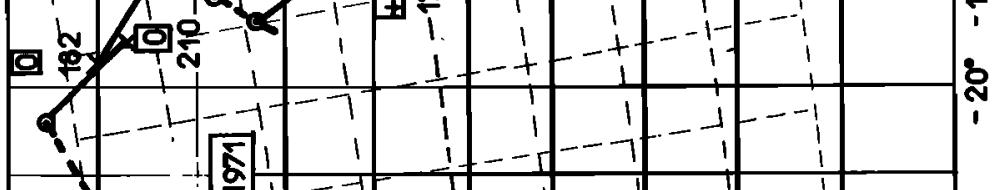

$\frac{1}{8}$

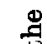

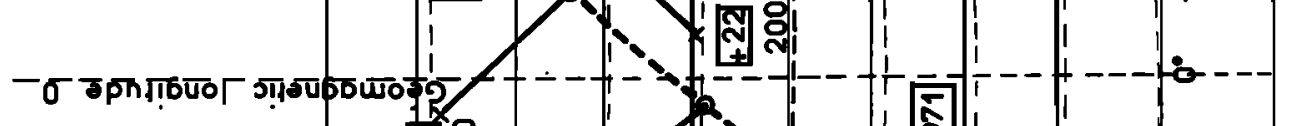

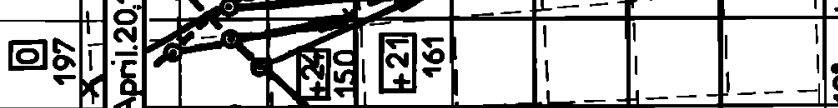

武?

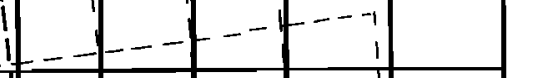

논

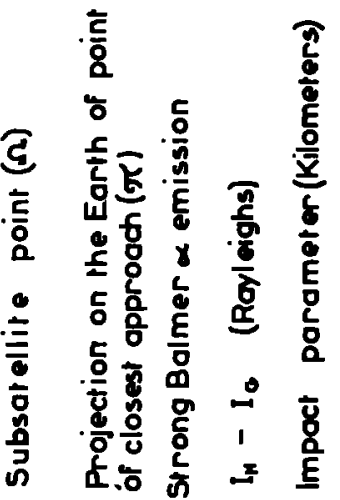

이요 
We would like to tentatively suggest that this Balmer $\alpha$ emission is due to protons either precipitated from the inner belt or accelerated from the ionospheric plasma.

Acknowledgments. This work was supported under CNES contracts 012 (1966), 268 (1969), 210 (1970), 201 (1971), and 201 (1972).

The Editor thanks B. A. Tinsley and another referee for their assistance in evaluating this paper.

\section{REFERENCES}

Blamont, J. E., and A. C. Levasseur, Preliminary results on an $\mathrm{H} \alpha$ map of the Gum Nebula obtained with the D2A satellite, Proceedings of the Gum Nebula and related problems, NASA GSFC X 683-71-375, p. 220, 1971.

Blamont, J. E., S. Cazes, J. Charrat, C. Emerich, C. Laurent, and A. C. Levasseur, Premiers résultats des expériences placées a bord du satellite D2A, internal report, Sẹvice d'Aéronomie du Centre National de la Recherche Scientifique, Verrières le Buisson, 1971.

Carruthers, G. R., Apollo 16 far ultraviolet camera/ spectrograph: First observations, Science, 177, $788,1972$.

Chamberlain, J. W., Planetary coronae and atmospheric evaporation, Planet. Space Sci., 11, 901, 1963.

Javid, K. H., Die Mitte Rand, Variationen der Balmerlinien $\mathrm{H} \alpha, \mathrm{H} \beta$ auf der Sonnescheibe, $Z$. Astrophys., 53, 37, 1961.

Evans, D. S., Photometric observations of $\mathrm{H} \alpha$ in the solar spectrum, Mon. Notic. Roy. Astron. Soc., 100, 156, 1940.

Fishkova, L. M., and G. V. Markova, Variations in the intensity of the line $\lambda 6562 \mathrm{~A}$ in the spectrum of the nightglow of the sky, Dokl. Akad. Nauk SSR, 154, 799, 1960.
Ingham, M. F., The nightglow spectrum, 2, $\mathrm{H} \alpha$ radiation in the night sky, Mon. Notic. Roy. Astron. Soc., 124, 523, 1962.

Ingham, M. F., Observations of the night sky $\mathrm{H}_{\alpha}$ emission line, Publ. de l'Observatoire de Haute Provence, 10, 261, 1968.

Johnson, H. M., Mem., Mt. Stromlo Observ., 3, 15,1960 .

Levasseur, A. C., Compte rendu d'observations à l'Observatoire de Haute Provence, internal report, Service d'Aéronomie du Centre National de la Recherche Scientifique, Paris, 1969.

Levasseur, A. C., Initial results on Balmer $\alpha$ observations on board D2A satellite, paper presented at 15th Cospar Symposium, Madrid, 1972.

Prokundina, V. S., Observations of the line $\lambda 6562$ A in the night airglow spectrum, Spe ${ }^{r}$ ' F/ler' chemical and Radar Researches of

Airglow, 1, pp. 43-66, 1959.

Sheglov, P. V., The concentration of night airglow $\mathrm{H} \alpha$ emission towards the ecliptic, Sov. Astron. $A J, 2,289,1964$.

Smith, L. L., F. E. Roach, and R. W. Owen, The absolute photometry of the zodiacal light, Planet. Space Sci., 18, 207, 1965.

Tinsley, B. A., Initial results of geocoronal Balmer $\alpha$ observations, Planet. Space Sci., 15, 1757, 1967.

Tinsley, B. A., Variations of Balmer $\alpha$ emission and related hydrogen distributions, Space Res., 10, 582,1970 .

Tinsley, B. A., and R. R. Meier, Balmer $\alpha$ distributions over a solar cycle: Comparison of observation with theory, J. Geophys. Res., $76,1006,1971$.

Vidal-Madjar, A., and J. L. Bertaux, A calculated hydrogen emission in the exosphere, Planet. Space Sci., 20, 1146, 1972.

(Received October 27, 1972; accepted January 26, 1973.) 